\title{
Stock Control through Video Surveillance in Logistics
}

\author{
Mariarosaria Carullo ${ }^{3}$, Gianluca Cavaliere ${ }^{3}$, Aniello De Prisco ${ }^{2}$, \\ Michele Di Capua ${ }^{3}$, Alfredo Petrosino ${ }^{1}$, Donatella Padovano ${ }^{3}$, Gennaro $\mathrm{Nave}^{3}$, \\ and Daniele Ruggeri ${ }^{2}$ \\ 1 Department of Science and Technology, University of Naples Parthenope, \\ Centro Direzionale Isola C4, 80143 Napoli, Italy \\ 2 MagSistem srl, Zona Industriale, 81030 Gricignano di Aversa (Caserta), Italy \\ 3 Unlimited Software srl, Centro Direzionale Isola F/11, 80143 Napoli, Italy
}

\begin{abstract}
The transport sector has certainly witnessed the latest developments in information and communication technologies (ICT). In this context the objective of the CPILOS project is to develop a technological platform, based on IT infrastructures and services, that can support critical processes, like secure tracking and tracing of goods, using video surveillance facilities with radio-frequency identification (RFID) support. More specifically, the project involves the study and implementation of an integrated platform for quality control of goods, particularly perishable food coming from China and intended to be distributed in the Italian and European consumer markets (usually not controlled at the source).
\end{abstract}

Keywords: Stock Control, Video Surveillance, Logistic, RFID, Tracking, Anomaly Detection.

\section{Introduction}

In recent years there have been important advances in the transport of perishable goods [8], both from the point of view of the improvement of established technologies and on the development of new solutions, all as a result of numerous factors that are shaping international economic scenario, from the market globalization to the trade internationalization. The rationalization of the transport system and the introduction of more effective and efficient logistics services that meet the needs of the different entities involved in the sector (producers, distributors, transporters), for perishable products not only translates into a reduction in transport costs and then the acquisition of value-added also by the production, but especially in improving the quality of products imported or exported from emerging economies such as China. Time, speed, accuracy of transactions, information flows and delivery processes are essential factors for industries that deal with products with a short life cycle and / or shelf life, especially products intended for human consumption.

The existing ICT systems and services built to support the operations of planning, management and control of distribution logistics and intermodal transport

A. Petrosino (Ed.): ICIAP 2013, Part II, LNCS 8157, pp. 740-748, 2013.

(C) Springer-Verlag Berlin Heidelberg 2013 
does not seem to be able to meet the growing needs of intermodal transnational transport [8]. As distribution centers and container parks grow and the number of incoming and outgoing trucks increases, the opportunities for theft, fraud, injury, and costly inefficiencies also grow. Modern container parks are a good example of the security issues related to the monitoring of acres of containers and drivers coming and going at all hours of the day and night.

In the CPilos project we propose an automated video system that is able to monitor the packages, burns RFID data onto the video image, and indexes the video stream according to the RFID information. The tag-read data and video images are used to verify shipment and provide electronic proof of delivery. The solution provided aims to design an efficient system for Small and Medium Enterprises in logistics that have to protect all shipments in transit against theft. The system can be used to monitor the movement of cargo in freight terminals for later reviewing in looking for ways to improve the efficiency and safety of personnel, truck traffic, loading procedures, and other activities.

\section{The Layout}

From a topological point of view, the CPILOS platform is physically organized in operational areas called Pilos Park, (Figure 1), each equipped with one or more functional spaces called Pilos Box.

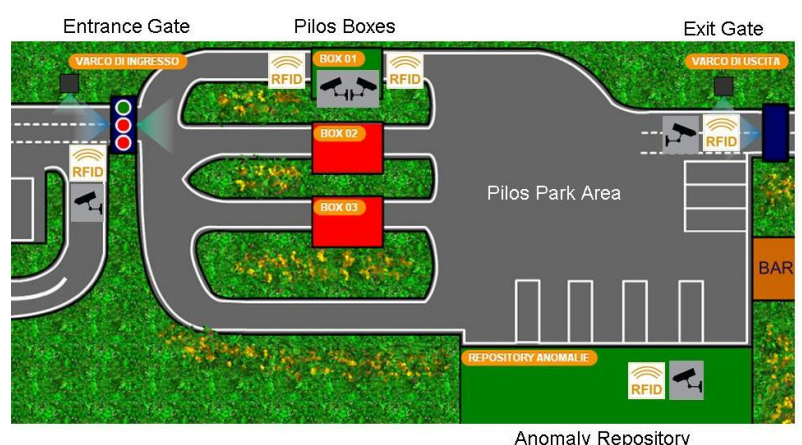

Fig. 1. Example of a typical Pilos Park with 3 Pilos Box (only one active)

A Pilos Box is an area of handling and storage of goods, placed near the port terminals and with an integrated intermodal transport system. In each operational zone a CCTV system with several cameras are installed, to cover the whole movement area, and also each Pilos Box is monitored by a dedicated camera. The Pilos Box includes facilities management, information and computer technology infrastructure, as well as warehouses, where different activities on products can be carried out. For example, in the case of perishable food products, analysis of organoleptic aspects quality, in not destructive mode, are carried 
out. The collected data are then stored on a centralized database. The Pilos Box is also equipped with an anomalies repository, where the goods can be stored, in case of detected problems. Finally, an integrated identification system of trucks and freight, based on RFID technology, is available to register and follow, in real time, the different operations involved in goods management. Each Pilos Park is connected to a network infrastructure controlled by a central control room (CPILOS control center). The control room, connected to the whole system, formed by all the Pilos Box and by the identification systems installed at the entrance and exit of the operational areas, plays an important role in data storage, and, through appropriate processing, produces analysis of historical and forecasted data. A flexible rule engine is provided, at the business logic level, in order to create relationships between actions and detected events, i.e., activate an alarm in case of not authorized human presence in a certain Pilos Park area, or unwanted freight movement inside a Pilos Box. Particular attention was paid to the so-called tracking and tracing activity, in order to address important and complex issues, like parallel imports, theft, and counterfeiting of products, and access management with multi-modality identification of users through the integration of biometric identification systems, and advanced video surveillance systems.

\section{Platform Design and Architecture}

The CPILOS platform has been designed to be modular and flexible in order to be open to future extensions of development. These features are of extreme importance not only to implement the platform in other geographical contexts, but also to allow adding, with the appropriate technological equipment, quality controls for different types of products, typically coming from China, such as textiles products. The platform offers an added value at two levels: 1) quality information of goods shipped, and 2) repository of statistical data.

The CPILOS platform is characterized by the coexistence of several subsystems that need to communicate with each other, regardless of the nature of the offered services and features. The single software components identified within the CPILOS platform are: Video surveillance system; Tracking and tracing system; Goods system management and identification via RFID; Data Storage; Systems for input/output events; and Business Logic Unit. In Figure 2 the different layers and components relationships are illustrated.

The interoperability issues concern the use of different communication protocols and data formats. The proposed solution is based on an integration architecture model built upon an ESB (Enterprise Service Bus) layer [12]. The adopted ESB is a distributed ESB open source software, MULE, that can be connected to different CPILOS Parks via an arbitrary set of configurable connectors, abstracting such systems and managing the exchange of messages between them and their processing. The key advantage of an ESB is that it allows different applications to communicate with each other by acting as a transit system for carrying data and events between applications within the CPILOS architecture or across the Internet. 


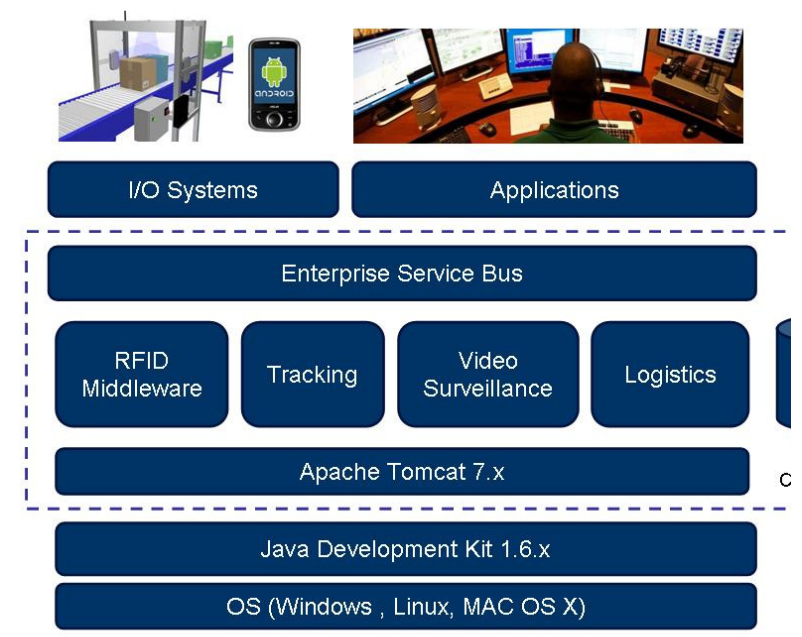

Fig. 2. Layers and basic components in the CPILOS architecture

In the CPILOS view the ESB must provide a distributed message-oriented architecture, supporting traditional Enterprise Application Integration features, such as messages and events routing and transformation, within the context of integration based upon 'business services' and XML messages.

The term 'service' acts as a reminder that the ESB is often seen as a central (indeed, the central) component of the service-oriented architecture (SOA). The SOA enables chunks of enterprise functionality to be presented as 'services' to the ESB, which routes, transforms, and validates the XML inputs and outputs from these services.

In order to support integration across widely distributed logistics deployments, the ESB itself must be deployed as a genuinely distributed system. Specifically, there must be no requirement to route all messages through a central hub in order to apply routing and transformation rules to them, that would have severe performance implications for even small logistics deployments. This is a key requirement for the ESB, leaving the traditional 'hub and spoke' approaches to integration and enabling solutions to be implemented in a truly incremental fashion and gradually connected across the enterprise. One trend is to provide micro-hubs to which key services such as transformation are delegated.

\section{Video Surveillance System}

The Video Surveillance System employs techniques based on computational intelligence to detect and track human activities, in order to identify and extract normal and anomalous behavior [1 6$]$. Detection, location, and tracking of nondeformable objects in the scene is relatively easy. However, segmentation and 
tracking of the human figure, within an unstructured environment, is a challenging task. The methodology of attentive vision systems has recently emerged as a key response to a large number of problems that arise in this field. These systems, if properly trained, appear to be able to identify directly and independently of the type and nature of the image at hand, the specific visual elements on which the expert's analysis focuses. In this project we have adopted a biologically inspired model in which the mechanisms of attentive vision are used to locate objects that attract the user attention with respect to some salient features, such as, for example, color, movement, and shape. In particular, the characteristics extracted from each frame serve as a basis to detect motion within the scene. We have developed a technique for background subtraction in which the model of the background is automatically generated. The background model learns the variations in the scene, treating them as pixel trajectories over time. The innovative idea consists in exploiting the model of the learned background through a map of moving and stationary patterns, to detect the movement in the scene. The latter is instantiated by the objects which deviate from the model, and is used to selectively update the model. Among the objects detected as not belonging to the background, particular attention has to be given to the objects temporarily stopped into the scene, i.e., those moving objects detected as stopped and not belonging to the background. To detect these events, the dual background method has been exploited, that consists in building two background models: a long-term model and a short-term model. The former models the scene background, while the latter models stopped objects. The actions detected into the scene need to be successively classified to recognize potential anomalous behaviors. Data collected, in terms of moving objects trajectories, have been employed to detect decision support systems based on machine learning techniques. In particular, we have developed methods for anomaly detection that allow to detect anomalies into the scene with high accuracy. The proposed methods are based on the self-organizing approach and the possibilistic approach.

\section{The Integrated Tracking and Tracing Component}

GPS Tracking is a process which involves the Global Positioning System satellites to locate a given object or person. By employing this system, logistic or local courier companies can tag the location of every vehicle in their fleet at any given time [9], with high precision and accuracy with respect to other forms of tracking.

The CPILOS platform includes a tracking and tracing system, with geographical information system facilities, in order to provide information on the physical location of goods in transit towards the Pilos Park area. The GPS localization is adopted, apart from the classical fleet management along the shipment, also inside the movement area (Pilos Park) that contains the Pilos Boxes. Custom geo-fenced areas (called geo-zones, see Figure 3) can be set up to provide arrival/departure notification events (fired along the ESB link). 


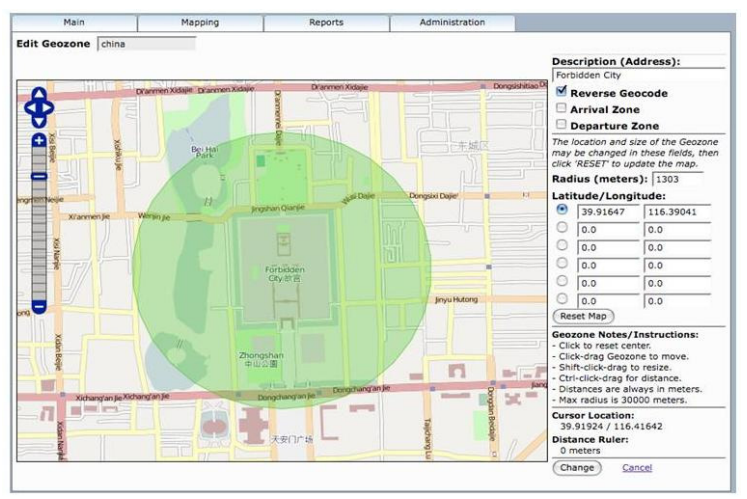

Fig. 3. A sample of geo-zone (green area) defined for arrivals/departures events

These events are then processed by the surveillance systems that can direct appropriate cameras to record the activity. Most GPS receivers have a standard accuracy of +/- 20 meters, or approximately +/- 3 meters if the GPS receiver incorporates the Wide Area Augmentation System. For fleet vehicle tracking purposes, +/- 20 meters often provides enough accuracy to determine geo-fence arrival/departure, and general location information. While having $+/-3$ meter accuracy can provide "parking space" accuracy (not just knowing that it is in the parking lot).

The tracking component of CPILOS is composed of common devices that send GPS data, over GPRS, about the position to a central server connected with the control room, that can track in real-time the location of the vehicles using digital maps. GPS are space-based radio positioning systems that provide 24-hour, 3-dimensional position, velocity, and time information to suitably equip users anywhere on the surface of the Earth [10]. The impact of these mobile technologies is more prevalent in contemporary sophisticated logistics which include multi-tiered suppliers, and manufacturers that are globally dispersed. Therefore, it is apparent that, with an increase of global integration and complex business networks, there is an imperative to develop network options beyond the boundaries of internal logistics. This brings new opportunities, as risks inherent with implementing new logistics systems are lowered. With particular reference to shipping lines, there has been an enduring issue with part of the global supply chain, called the first and the last mile. These terms refer to the stages of the delivery process where goods are collected from the shipper and delivered to the final customers in containers.

The technological solution adopted in CPILOS allows to create a real central independent control system, in order to reduce the cost of service and capable to control different types of GPS devices, to manage the access control and facilitate the integration with other systems. The software tracking platform used to implement the described above functionality is OpenGTS (Open Source GPS Tracking System), the first open source system that provides web-based 
services for querying and visualization of GPS data. Once the position information is processed by a mobile tracking device, it is transferred through GPRS to the CPILOS control room. This information can then be requested from a web site and displayed on a map offering an easy way to know exactly where the freight is. Moreover, using 'Fly with object' feature of Google Earth, it is possible to get an interesting view of a moving vehicle on Google Earth satellite imagery. OpenGTS comes with support for OpenLayers/ OpenStreetMap in addition to support for Google Maps, Microsoft Virtual Earth, and Mapstraction (which provides mapping support for MultiMap, Map24, MapQuest, and more). Within the OpenGTS framework, other mapping service providers can be easily integrated.

\section{System Management Identification and Control via RFID}

In the logistics context, RFID (Radio Frequency Identification) provides benefits in two ways. On the one hand it supports the coordination of the physical flow of goods and related value added information services, like tracking and tracing, condition monitoring, and supply chain event management, but also security related services. On the other hand it improves operational efficiency for traditional logistics processes like transportation control and asset management. RFID middleware can be considered the cornerstone of non-trivial RFID deployments [7] in complex heterogeneous logistics environments, comprising multiple readers, applications instances, legacy ICT systems, as well as sophisticated business processes and semantics.

Software that implements any combination of these information flows can be conceived as an RFID middleware [1]. The CPILOS project integrates the use of RFID technologies for the monitoring and control of the goods and in particular for the identification of the containers and for the control of the cargo loading / unloading activities. This requirement involves the integration, within the platform, of the above mentioned middleware, connected, along the available ESB link, in a network with different readers. Starting from the identification codes from the TAG, this system allows to obtain all available information associated with "smart objects" and make this information available within the platform. An automated video system monitors the packages, burns RFID data onto the video image, and indexes the video stream according to the RFID information. The tag-read data and video images are used to verify shipment and provide electronic proof of delivery. Every time a tag is scanned on freight, system software calculates the relative tag location and directs the appropriate cameras to record the activity. A data marker enables easy retrieval of the video record. To reduce video storage needs, just four to five seconds of video is recorded.

The RFID integration in video surveillance system allows to:

- Prevent loss, enabling freight and warehousing companies to track warehouse deliveries and loading activities, ensure trailers are fully unloaded, document 
fraudulent worker compensation claims for accidents that never happened, and make better decisions on how to improve security around the Pilos Park.

- Improve operations, by monitoring the movement of cargo in freight terminals for later reviewing in order to improve the efficiency and safety of personnel, truck traffic, loading procedures, and other activities.

The CPILOS RFID layer is built upon open source software RIFIDI, a system that allows both rapid prototyping and development of RFID applications for the production, which require advanced features, such as high performance, scalability, etc.. RIFIDI is able to emulate different RFID systems and it allows the virtual creation of an RFID-based scenario, while ensuring that the software created for this purpose will work also in the real world.

\section{Conclusions}

After analyzing in detail the various components of the CPILOS platform, it may be useful to summarize the overall architecture design and provide the technical details of the development process. First, we must consider the presence of the enterprise service bus system as the backbone of all communications, through which all data and events that cross the platform. The ESB allows the connection between different remote Pilos Park, in a flexible way, providing a centralized point of observation, that takes the form of the CPILOS control room. Classified events inside the logistic platform (motion detection, tag reading, geofenced events), are filtered and processed by the ESB, and then fired to the Business Logic Unit, which activate the correct tools to manage the different scenarios. Also video cameras data flow (streaming) is directly routed through the enterprise service bus.

Acknowledgment. This research was fully supported by the Italian Ministry of Education, University and Research (MIUR) under grant GPS - DM29045.

We are indebted to F. Camastra, A. Ciaramella, A. Ferone, A. Maratea and A. Staiano for their contribution to the project.

\section{References}

1. Breitenstein, M.D., Reichlin, F., Leibe, B., Koller-Meier, E., Gool, L.V.: Robust tracking-by-detection using a detector confidence particle filter. In: ICCV (2009)

2. Cuntoor, N.P., Yegnanarayana, B., Chellapa, R.: Activity modeling using event probability sequences. IEEE Trans. on Image Process. 17(4), 594-607 (2008)

3. Ess, A., Leibe, B., Schindler, K., van Gool, L.: A mobile vision system for robust multi-person tracking. In: CVPR (2008)

4. Hamid, R., Maddi, S., Johnson, A., Bobick, A., Essa, I., Isbell, C.: A novel sequence representation for unsupervised analysis of human activities. Artificial Intelligence 173, 1221-1244 (2009)

5. Kuettel, D., Breitenstein, M.D., Van Gool, L., Ferrari, V.: What's going on? Discovering spatio-temporal dependencies in dynamic scenes. In: CVPR (2010) 
6. Pal, S.-K., Petrosino, A., Maddalena, L. (eds.): Handbook on Soft Computing for Video Surveillance. Chapman \& Hall/CRC (2012)

7. Kefalakis, N., Leontiadis, N., Soldatos, J., Donsez, D.: Middleware Building Blocks for Architecting RFID Systems. In: Granelli, F., Skianis, C., Chatzimisios, P., Xiao, Y., Redana, S. (eds.) MOBILIGHT 2009. LNICST, vol. 13, pp. 325-336. Springer, Heidelberg (2009)

8. Leal, E., Pérez Salas, G.: Logistic platforms: conceptual elements and the role of the public sector. FAL Bullettin 274(6) (2009)

9. Mintsis, G., Basbas, S., Papaioannou, P., Taxiltaris, C., Tziavos, I.N.: Applications of GPS technology in the land transportation system. European Journal of Operational Research 152(2), 399-409 (2004)

10. Mondragon Coronado, A.E., Lalwani, C.S., Mondragon Coronado, E.S., Mondragon Coronado, C.E.: Facilitating multimodal logistics and enabling information systems connectivity through wireless vehicular networks. Int. J. Production Economics 122, 229-240 (2009)

11. Zarokostas, N., Dimitropoulos, P., Soldatos, J.: RFID Middleware Design for enhancing traceability in the Supply Chain Management. In: 18th IEEE Personal Indoor and Mobile Radio Communications, Athens, Greece (2007)

12. Schmidt, M.T., Hutchison, B., Lambros, P., Phippen, R.: The enterprise service bus: Making service-oriented architecture real. IBM Systems Journal 44(4), 781-797 (2005) 